\title{
Spontaneous recanalisation of side branches occluded during percutaneous transluminal coronary angioplasty
}

\author{
M F SHIU, A SINGH \\ From the University Department of Cardiovascular Medicine, Queen Eizabeth Hospital, Birmingham
}

SUMmaRY Percutaneous transluminal coronary angioplasty was performed in a 56 year old man with postinfarction angina. During an otherwise uncomplicated dilatation of a left anterior descending artery with a $70 \%$ stenosis two diagonal branches, each measuring $1.5 \mathrm{~mm}$ in diameter, were occluded. The occlusions were not associated with any adverse clinical effects, though there was a small rise in plasma creatine kinase concentration. The patient became free of angina two weeks after angioplasty, and follow up angiography showed spontaneous reappearance of the occluded side branches. Redistribution of atheromatous material and its later reabsorption may have been the mechanism for the initial occlusion and later reappearance of the vessels.

Side branch occlusion is a recognised complication of percutaneous transluminal coronary angioplasty. Side branches originating directly in the stenotic segment have been shown to be particularly at risk, and it is often a contraindication to coronary angioplasty. ${ }^{1}$ In the absence of a systematic follow up study of these occluded side branches, the assumption is that these vessels would remain permanently blocked. We report the spontaneous recanalisation of two diagonal branches occluded during an otherwise uncomplicated percutaneous transluminal coronary angioplasty of a left anterior descending artery.

\section{Case report}

A 56 year old man with severe postinfarction angina unresponsive to medical treatment was investigated with a view to surgery. There were no detectable risk factors apart from previous heavy cigarette consumption of 60 per day. A resting electrocardiogram showed pathological $Q$ waves in inferior leads. Stress testing on a bicycle ergometer showed $1 \mathrm{~mm} \mathrm{ST}$ depression in leads II, III, and V4-V6 associated with

Requests for reprints to Dr M F Shiu, Department of Cardiovascular Medicine, University of Birmingham, Queen Elizabeth Hospital, Birmingham B15 2TH chest pain at $100 \mathrm{~W}$. Stress thallium imaging showed a fixed inferior apical defect and reversible ischaemia in the diagonal and lateral territories. Cardiac catheterisation 18 months after infarction showed impaired left ventricular function with inferior hypokinesis and an ejection fraction of $34 \%$. The right coronary artery was occluded near its origin with no antegrade or cross filling of distal branches. The circumflex system was non-dominant with only minor irregularities. The proximal left anterior descending branch was ectatic in part with a concentric stenosis of approximately $70 \%$ over a segment involving the origins of a septal branch and two diagonal branches, each measuring approximately $1.5 \mathrm{~mm}$ in diameter (Fig. 1a).

Percutaneous transluminal coronary angioplasty was performed using the femoral approach and a 3.7 $\mathrm{mm}$ balloon with an 0.016 inch flexible tipped wire Schneider-Medintag. The stenosis in the left anterior descending artery was crossed without difficulty. The transstenotic pressure gradient was $46 \mathrm{~mm} \mathrm{Hg}$ falling to $6 \mathrm{~mm} \mathrm{Hg}$ after three inflations at 8 atmospheres, each lasting $40 \mathrm{~s}$. The procedure was not accompanied by chest pain, arrhythmia, or hypotension. Immediate repeat arteriography showed an improvement of the lesion with a $25 \%$ residual stenosis. There was non-opacification of the two diagonal branches (Fig. 


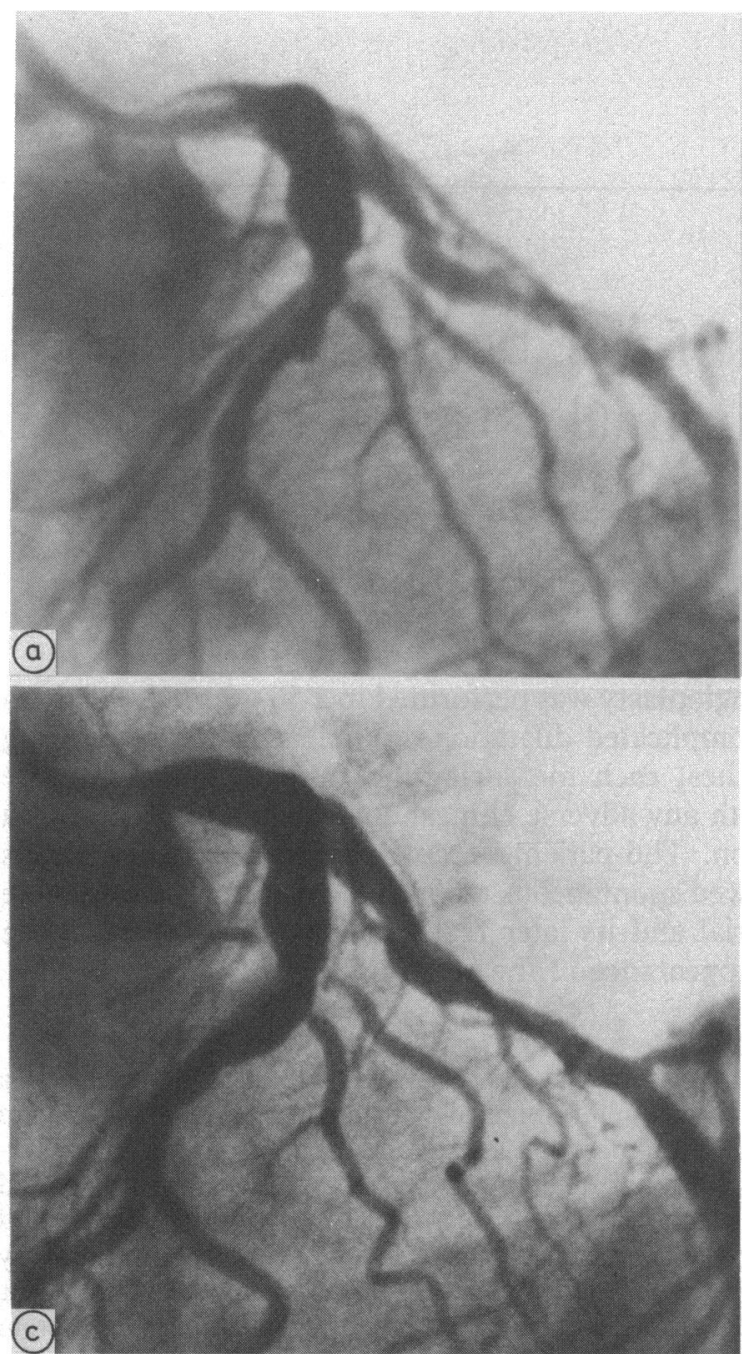

lb). The patient was given 10000 units of heparin intravenously at the start of the procedure but no other anticoagulant. Aspirin was given on the day before the procedure and continued to be taken at 300 $\mathrm{mg}$ daily. Intracoronary nitrate $200 \mu \mathrm{g}$ was given before balloon passage and after each inflation up to a total dose of $1 \mathrm{mg}$.

The hospital stay was uneventful. A repeat electrocardiogram showed no changes over the next 48 hours. Plasma creatine kinase concentration rose from a preoperative value of $180 \mathrm{IU} / 1$ to a maximum of 302 IU/1 24 hours after the procedure with traces of cardiac isoenzymes. After discharge from hospital the patient reported mild anginal chest pain for two weeks

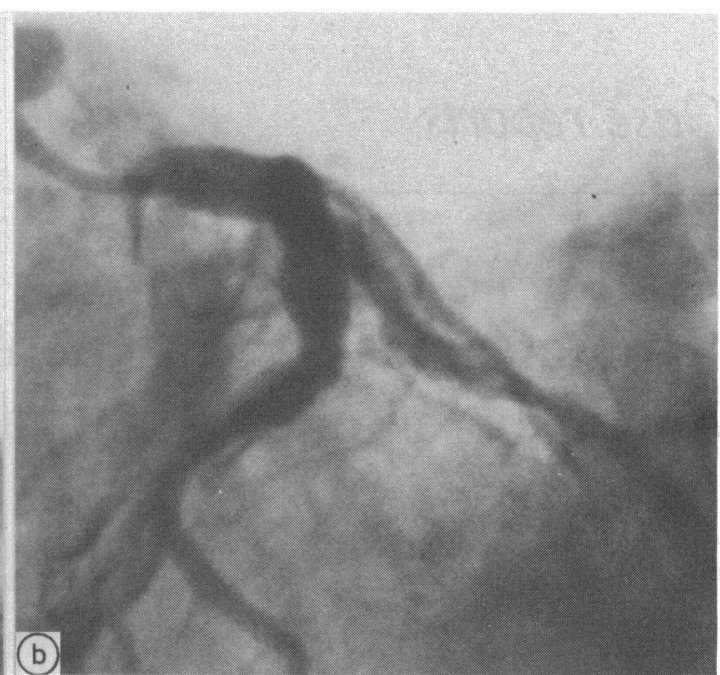

Figure Left coronary arteriograms in the left anterior oblique (cranial) view showing two diagonal vessels, each measuring 1.5 $\mathrm{mm}$, involved in the left anterior descending artery stenosis (a) before angioplasty, (b) immediately after angioplasty, when both diagonal branches were occluded at the origin, and (c) six months later, when both diagonal branches had fully recanalised.

after operation. From the third week his symptoms disappeared despite increasing activity and withdrawal of all antianginal medications other than nifedipine $10 \mathrm{mg}$ three times daily. Repeat exercise testing at three and six months showed an increased maximum capacity of $120 \mathrm{~W}$ with no subjective chest pain and no diagnostic ST-T changes in the anterior chest leads. Repeat thallium stress testing showed no reversible perfusion defects in the areas subtended by the anterior descending and diagonal vessels. A reperfusion image at four hours showed a fixed defect in the inferior region as before. Follow up coronary arteriography performed as a routine at six months showed no progression of the left anterior descending 
stenosis. The two previously occluded diagonal branches were patent and appeared unchanged since before coronary angioplasty (Fig. lc).

\section{Discussion}

The loss of a side branch as a result of coronary angioplasty is an important consideration in the selection criteria of patients for this procedure. ${ }^{2}$ Most operators would avoid lesions involving side branches $>2 \mathrm{~mm}$ in diameter since the occlusion of such vessels is likely to result in a clinical myocardial infarction. Meier et al have shown that side branches are rarely occluded unless they arise directly from the stenotic segment of the main vessel, when their origins are often diseased. ${ }^{1}$ In such instances the occlusion rate was about $14 \%$. The diagonal branches in our patient belonged to this category and were occluded as a result of an otherwise uncomplicated angioplasty of the main vessel.

The reappearance of the occluded side branches is the main interest in this report. Such spontaneous reappearances have not been previously documented but may have escaped notice if not specifically looked for at the time of follow up angiography. Spontaneous reappearance of vessels occluded in the course of the natural history of coronary artery disease is rare. ${ }^{3}$ The mechanism of vessel occlusion during angioplasty is probably different from the natural disease. ${ }^{45}$ Meier et al observed angiographic evidence of coronary dissection in a high proportion of their patients with branch occlusion and concluded that involvement of side branches by the dissection was the prime mechanism. ${ }^{1}$ Other possibilities include coronary spasm, thrombosis, and redistribution of atheromatous plaque material. 5 In our patient, careful review of the angiograms showed none of the usual features of coronary dissection such as an intimal cleft or vessel wall staining. Although dissection could not be excluded, it was probably unlikely. Similarly, coronary spasm cannot be ruled out, but the abrupt loss of both vessels at the point of their orgins was more suggestive of an organic obstructive lesion. Such obstructions may be due to thrombus or atheromatous material. The last can be explained by the redistribution of atheromatous material from the adjacent left anterior descending vessel wall. The fact that the origins of the side branches were directly involved in the stenotic lesion meant that total occlusion can result from even a small amount of sequestrated debris. Despite the transient occlusion of two side branches our patient had a good clinical response to the angioplasty procedure. It is not possible to be dogmatic about whether the branch occlusion resulted in myocardial necrosis, but on balance it seemed unlikely.

The modest rise in cardiac enzymes with only traces of cardiac isoenzyme is often seen in otherwise uncomplicated cases. Our patient experienced no chest pain during his hospital stay, and there were no other clinical features of myocardial infarction. Our hypothesis is that occlusion was temporary and that early spontaneous recanalisation occurred leading to the improvement of anginal symptoms in the three weeks after the angioplasty procedure.

The most likely explanation for spontaneous recanalisation of the branches is reabsorption of atheromatous debris. Such clearing up of intraluminal debris has been suggested as one of the mechanisms by which coronary lesions continue to improve weeks after coronary angioplasty. ${ }^{4}$ It is not known what, if any, pharmacological agents would enhance this process. Oral anticoagulation has been shown to have no effect on the results of coronary angioplasty, and it was not used in this case. ${ }^{6}$ Our patient had the standard regimen of systemic heparinisation during operation, and treatment with aspirin $300 \mathrm{mg}$ daily was started on the day of the procedure and continued afterwards. Further observations on spontaneous recanalisation of occluded side branches may help in our understanding of the healing process after vessel injury at coronary angioplasty.

\section{References}

1 Meier B, Gruentzig AR, King SB, et al. Risk of side branch occlusion during coronary angioplasty. $A m \mathcal{F}$ Cardiol 1984; 53: $10-4$.

2 Cowley MJ, Vetrovec GW, Wolfgang TC. Efficacy of percutaneous transluminal coronary angioplasty: technique, patient selection, salutary results, limitations and complications. Am Heart f 1981; 101: 272-80.

3 Bruschke AVG, Wijers TS, Kolsters W, Landmann J. The anatomic evolution of coronary artery disease demonstrated by coronary arteriography in 256 nonoperated patients. Circulation 1981; 63: 527-36.

4 Block PC. Percutaneous transluminal coronary angioplasty. AFR 1980; 135: 955-9.

5 Holmes DR Jr, Vlietstra RE, Mock MB, et al. Angiographic changes produced by percutaneous transluminal coronary angioplasty. Am $\mathcal{F}$ Cardiol 1983; 51: 676-83.

6 Thornton MA, Gruentzig AR, Hollman J, King SB, Douglas JS. Coumarin and aspirin in prevention of recurrence after transluminal coronary angioplasty: a randomized study. Circulation 1984; 69: 721-7. 